\title{
Probing the interaction mechanism between Direct Red 80 and Human serum albumin: studies at the molecular level
}

\author{
Yan Song ${ }^{1}$, Yuzhi Shi $^{2 *}$, Kejia Zhang ${ }^{2}$ \\ 1. School of Water Conservancy and Environment, University of Jinan, Jinan, Shandong Province,250022 \\ 2. Water Resources Research Institute of Shandong Province, Jinan,250014, China
}

\begin{abstract}
In recent years, azo dyes have received increasing attention due to their adverse effects on the environment and consumer health. However, the interaction mechanism between human serum albumin (HSA) and Direct Red 80 (DR80) is still unknown. The results showed that DR80 changed the secondary structure of HSA and made HSA skeleton loose and stretch. In addition, DR80 quenched the endogenous fluorescence of HSA by static quenching and changed the microenvironment of Trp in form of the hydrophobicity increased and the polarity decreased. Molecular docking results indicated that DR80 bound to the interface of three $\alpha$ helical domains of HSA, hence, the changes in HSA structure and conformation was the main reason for the decline of its esterase activity. This work was done to illuminate the binding mechanism of DR80 and HSA, and to provide a different way for screening the low toxic dye at the molecular level.
\end{abstract}

\section{Introduction}

It is well known that the absorption, distribution, metabolism and excretion of various ligands in the body are affected by the mechanism of protein-receptor interactions in blood[1]. In recent years, the field of dyeprotein interaction has become a common concern of chemistry, environmental science and clinical medical workers. Among them, azo dyes have received increasing attention due to their adverse effects on the environment and consumer health[2]. In addition, according to the studies performed by T.O. Hushcha[3], serum albumin interacts with low molecular weight dyes to cause conformational changes, which affects the secondary and tertiary structure of albumin. Therefore, it is necessary to investigate the interaction of dyes and drugs to serum albumin.

Direct Red 80 (DR80) is a typical naphthalene sulfonated azo dye, and its application range has been extended from the dyeing of the original textile to paint, plastic, paper, leather, optoelectronic communication, clinical medicine, etc. What's more, DR80 has been used as a food colorant, cosmetic ingredients, medical disinfectant and insecticide. However, DR80 has been found carcinogenic and mutagenic, such dyes are extremely difficult to biodegrade in the natural environment and through a series of activation effects, it becomes an inducer of cancers.

HSA, a protein widely present in plasma, accounts for about $60 \%$ of total plasma protein, and has important physiological functions for transporting fatty acids, bile pigments, amino acids and other exogenous substances. Pollutants enter the human body mainly through the blood to the whole body, there is evidence that the interaction between dyes and HSA can change the structure and function of HSA itself, causing its loss of oxygen carrying capacity, which will have adverse effects on human organs[4].

In this paper, the binding mechanism between DR80 and HSA was studied in respects of the protein size, and the effect of DR80 on the structure and function of HSA. This work aims to illuminate the binding mechanism of DR80 and HSA, and to establish a different way for screening the low toxic dye at the molecular level.

\section{Materials and methods}

\subsection{Test materials}

The structural formula of DR80 is shown in Fig.1. HSA was purchased from Sigma, USA, and stored at $4^{\circ} \mathrm{C}$. The drugs used in the experiment were of analytical pure. 


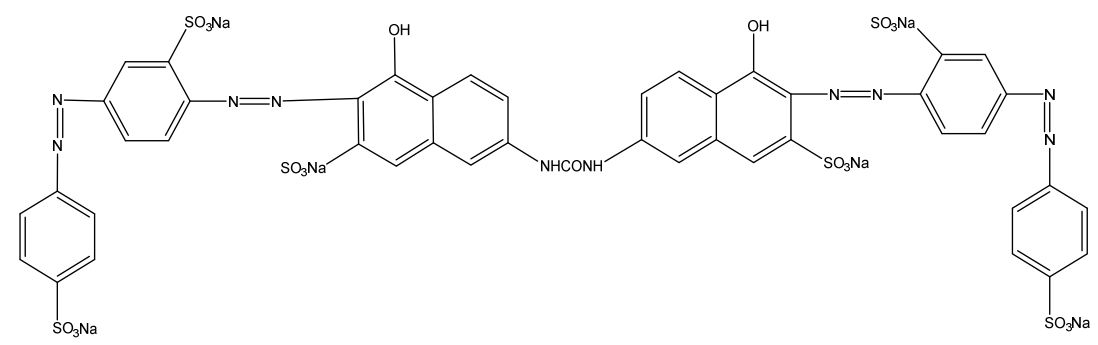

Fig.1 Structure of Direct Red 80

\subsection{Experimental instrument}

In this study, the fluorescence spectra were measured using the F-4600 fluorescence spectrophotometer (Hitachi, Japan); The UV-vis absorption spectra were measured using a UV-2600 spectrophotometer (Shimadzu, Kyoto, Japan); The CD spectra were measured using a J-810 CD spectrometer (JASCO, Japan).

\subsection{Fluorescence analysis}

Fluorescence spectra were determined by a $1 \mathrm{~cm}$ quartz cell. The excitation wavelength was set at $280 \mathrm{~nm}$; the scanning range and scanning speed was set to $290-450 \mathrm{~nm}$ and $1200 \mathrm{~nm} / \mathrm{min}$, respectively; The excitation and emission slit widths were both $5 \mathrm{~nm}$; PMT (photo multiplier tube) voltage was $600 \mathrm{~V}$.

Synchronous fluorescence spectroscopy was performed between the excitation and emission wavelengths at a fixed wavelength interval $(\Delta \lambda=15 \mathrm{~nm}$ and $\Delta \lambda=60 \mathrm{~nm}$ ), with excitation wavelengths starting at 265 to $310 \mathrm{~nm}$ respectively.

\subsection{UV-vis analysis}

The UV-visible absorption spectra of the experimental system were scanned using a $1 \mathrm{~cm}$ quartz cell. The wavelength range was set to $190-650 \mathrm{~nm}$; the slit width was $2 \mathrm{~nm}$. The ultrapure water was used for zero adjustment and a solution containing no HSA but containing the same concentration of DR 80 and PB buffer was used as a reference.

\subsection{Molecular docking study}

Molecular docking simulations were performed on MOE (version: 2009; Chemical Computing Group, Canada) software. Remove all water molecules from 1BJ5 before docking and add hydrogen atoms. The Site finder module was used to find possible docking locations and the MOEDock module was set to calculate the docking process.

\subsection{Esterase activity}

Using a UV-2600 spectrophotometer with a $1 \mathrm{~cm}$ quartz cell, adding $1 \mathrm{~mL}$ of the experimental system solution, 2 $\mathrm{ml}$ of p-nitrophenyl acetate solution, mixing and scanning at $400 \mathrm{~nm}$ for the initial and $10 \mathrm{~min}$ absorption values respectively. The reaction results were subjected to a pseudo first-order reaction rate constant analysis, and the esterase activity of HSA was calculated according to the following formula:

$$
\text { Inhibition } \operatorname{rate}(\%)=\Delta \mathrm{b}_{1} / \Delta \mathrm{b}_{0}
$$

where $\Delta \mathrm{b}_{0}$ is the difference in absorption value of the system when no small molecule is added, $\Delta b_{1}$ is the difference in absorption value of the system when small molecules of different concentrations are added.

\section{Result and discussions}

\subsection{The effects of DR80 on the intrinsic fluorescence of HSA}



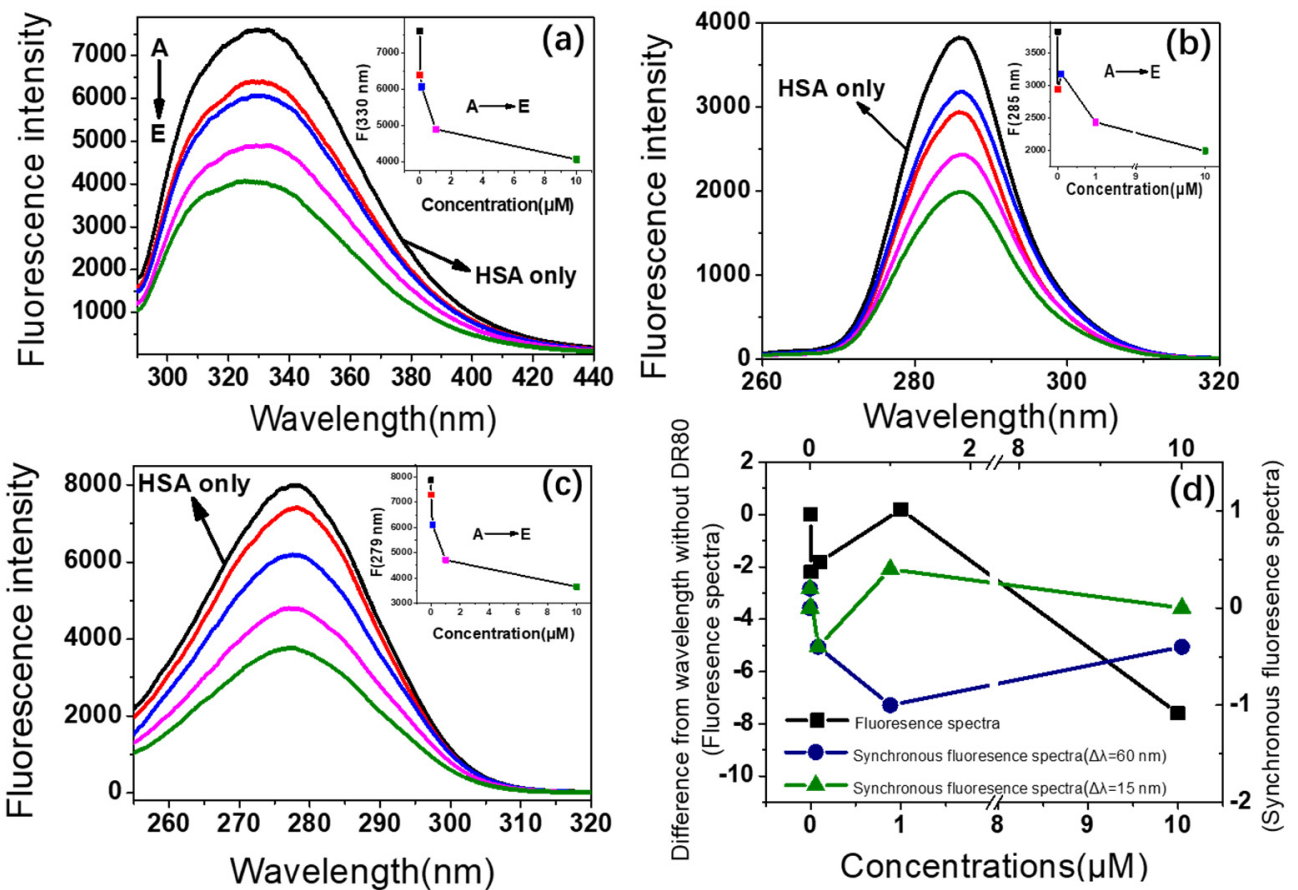

Fig.2 Effect of DR80 on HSA amino acid residue.

(a) Fluorescence spectra of HSA. (b) Synchronous fluorescence spectra $(\Delta \lambda=15 \mathrm{~nm}$ ) of HSA. (c) Synchronous fluorescence spectra $(\Delta \lambda=60 \mathrm{~nm})$ of HSA. (d) Difference in wavelength of fluorescence and synchronous fluorescence spectra from the sample without DR80.

Conditions: $\mathrm{T}=310 \mathrm{~K}, \mathrm{pH}=7.4$, [HSA $]=1 \mu \mathrm{M}$ $[\mathrm{DR} 80](\mathrm{A} \rightarrow \mathrm{E})=0,0.001,0.1,1,10 \mu \mathrm{M}$

HSA contains aromatic amino acids, including tyrosine(Tyr), tryptophan(Trp) and phenylalanine (Phe) residues, which makes it fluoresce at around $280 \mathrm{~nm}$, and the endogenous fluorescence intensity of HSA may change after interacting with small molecular[5]. Therefore, Trp plays an important role in the endogenous fluorescence of proteins. The fluorescence spectra are wildly used to provide information about the changes in the microenvironment of the HSA fluorophores[6]. Synchronous fluorescence spectra can reflect the detailed information about the microenvironment of Tyr and Trp when the wavelength interval $(\Delta \lambda)$ of excitation and emission wavelengths is set at 60 or $15 \mathrm{~nm}$. Fig.S1 displayed the molecular structures of Try, Trp and Phe.

Fig. 2 showed the corrected fluorescence and the synchronous fluorescence spectra of HSA in addition of DR80 at different concentrations. From Fig.2(a) we can see that the fluorescence intensity of HSA was quenched regularly with the increasing concentrations of DR80, indicating that interaction occurred between DR80 and the fluorophores of HSA. Fig.2(d) showed the difference in wavelength of fluorescence and synchronous fluorescence spectra from the control sample (without DR80), which indicated that a blue shift occurred in the fluorescence spectra of HSA. The blue shift of peak position indicated that DR80 changed the microenvironment of the HSA chromophore, making it more exposed and hydrophobic. In addition, due to the changes in protein structure may affect the endogenous fluorescence intensity of the protein, it can be deduced that the skeleton structure of the protein have also changed with the treatment of DR80.
The synchronous fluorescence spectra of HSA were shown in Fig.2(b/c). With the increasing concentrations of DR80, the synchronous fluorescence intensity of HSA decreased, which is consistent with the trend of fluorescence spectra. As can be seen from Fig.2(d), the synchronous fluorescence spectra of $\operatorname{Trp}(\Delta \lambda=60 \mathrm{~nm})$ had a slight blue shift while the synchronous fluorescence spectra of the Tyr $(\Delta \lambda=15 \mathrm{~nm})$ had no obvious wavelength change, demonstrating that the microenvironment of Trp changed as the hydrophobicity increases and the polarity decreases. 


\subsection{Effect of DR80 on the conformation and function of HSA}
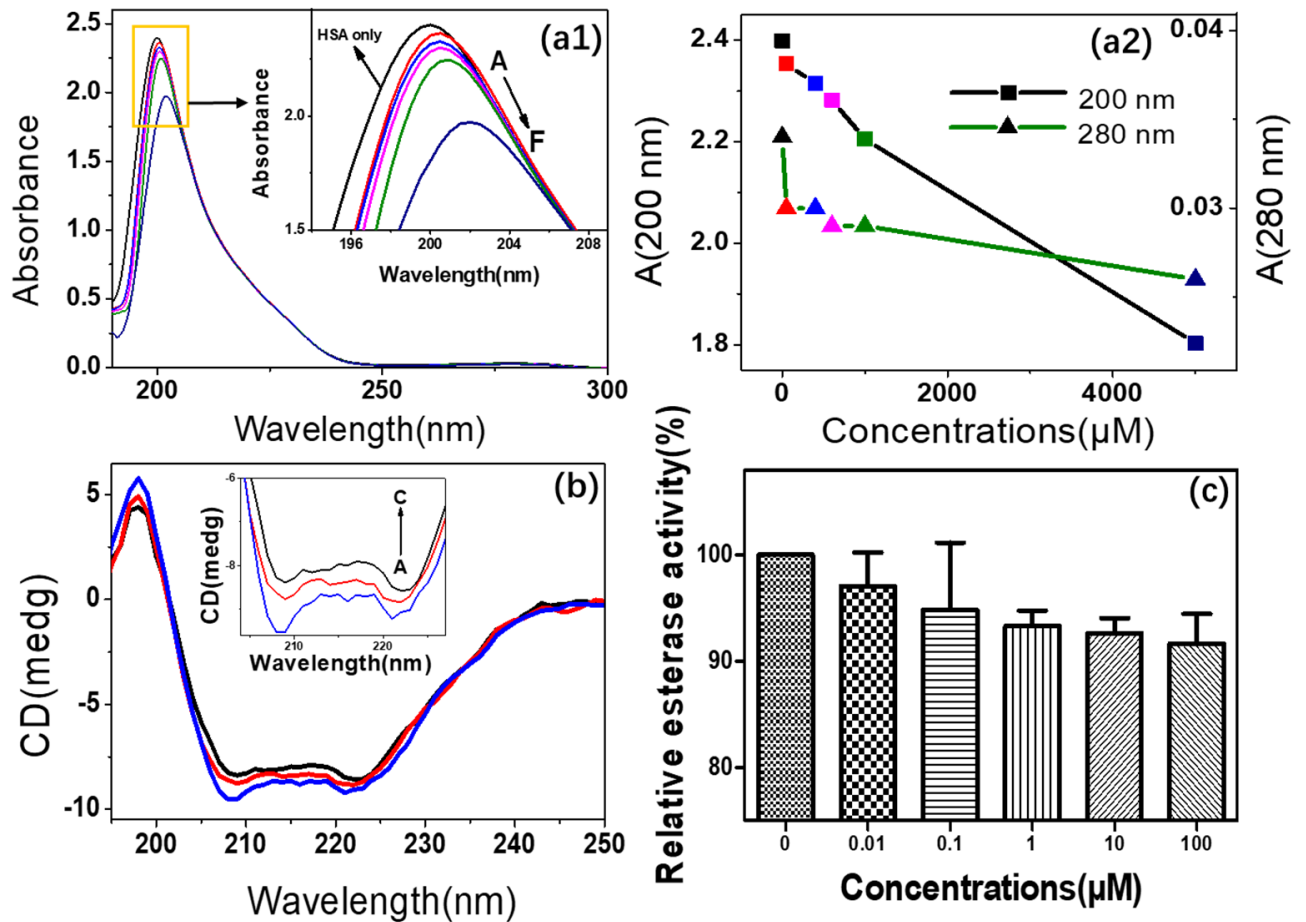

Fig.3 Effect of DR80 on the conformation and function of HSA.

(a1) UV-vis spectra; (a2) The scatter plot of UV-vis spectra of HSA with the addition of different concentrations of DR80; (b) CD spectra; (c) Relative esterase activity changes of HSA

Conditions: $\mathrm{T}=310 \mathrm{~K}, \mathrm{pH}=7.4,[\mathrm{HSA}](\mathrm{a} / \mathrm{b})=1 \mu \mathrm{M} ;(\mathrm{c})=500 \mu \mathrm{M}$

(a) $[\mathrm{DR} 80](\mathrm{A} \rightarrow \mathrm{F})=0,50,400,600,1000,5000 \mu \mathrm{M}$; (b) $[\mathrm{DR} 80](\mathrm{A} \rightarrow \mathrm{C})=1,100,600 \mu \mathrm{M}$;

(c) $[\mathrm{DR} 80]=0,0.01,0.1,1,10,100 \mu \mathrm{M}$.

Table.1 Effect of DR80 on the contents of HSA secondary structure content

\begin{tabular}{ccccc}
\hline \multirow{2}{*}{ DR80 Concentrations $(\boldsymbol{\mu M})$} & \multicolumn{3}{c}{ Secondary structure content in HSA (\%) } \\
\cline { 2 - 5 } & $\boldsymbol{\alpha}$-Helix & $\boldsymbol{\beta}$-Sheet & $\boldsymbol{\beta}$-Turn & Unordered \\
\hline 1 & 48.9 & 4.8 & 15.4 & 33.5 \\
100 & 43.7 & 5.5 & 15.1 & 36.8 \\
600 & 41.4 & 7.7 & 18.4 & 34.9 \\
\hline
\end{tabular}

Changes in skeleton structure. UV-visible absorption spectra were widely used to observe the changes of protein skeleton structure. As can be seen from the Fig.3a, the strong absorption band around $210 \mathrm{~nm}$ was produced from the $\pi-\pi^{*}$ electronic transitions of the peptide backbone $\mathrm{C}=\mathrm{O}$ which reflects the skeleton structure of proteins. The absorption band between $260-290 \mathrm{~nm}$ reflects the changes in the microenvironment of the aromatic amino acid residues Trp, Tyr and Phe residues.

With the addition of DR80, the intensity of the absorption peak at $210 \mathrm{~nm}$ decreased with a clear red shift, indicating that the skeleton structure of HSA was loosened and unfolded[7]. The absorption peak around $280 \mathrm{~nm}$ decreased with the increasing concentrations of DR80, which is consistent with the decreasing trend of fluorescence spectra.

Secondary structure contents determination. In order to further explore the effect of DR80 on HSA secondary structure, the circular dichroism spectra (CD) were investigated. The results after deducting the reference are shown in Fig.3(b). From Fig.3(b) we can see that there are two negative bands at $209 \mathrm{~nm}$ and $218 \mathrm{~nm}$, represented $\mathrm{n} \rightarrow \pi^{*}$ and $\pi \rightarrow \pi^{*}$ transition of $\alpha$-helix [8]. With the addition of DR80, the intensity of CD spectra showed an upward trend, which indicated that DR80 changed the secondary structure of HSA. The detailed changes of HSA $\alpha$-helix, $\beta$-sheet, $\beta$-turn, and unordered curling contents were calculated using the software provided by lamar.colostate.edu/ sreeram/Cdpro/main.html, and the results are displayed in Table.1. The content of HSA $\alpha$ helical structure decreased by $7.5 \%$, while the contents of $\beta$-sheet, $\beta$-turn and unordered curling increased by $2.9 \%$, $3 \%$ and $1.4 \%$ respectively, indicating that DR80 led HSA to misfold and partially denature.

Physiological function analysis. The relative activity of HSA was set to $100 \%$ and other groups with various concentrations of DR80 were calculated accordingly. As shown in Fig.3(c), all concentration groups of activity 
were lower than the group with treatment of DR80, and the activity of HSA decreased regularly with the increasing concentrations of DR80. This result indicated that the interaction of DR80 and HSA inhibited the activity of HSA and then further caused impact of its physiological function. According to the discussions above, we can see that DR80 changed the conformation and secondary

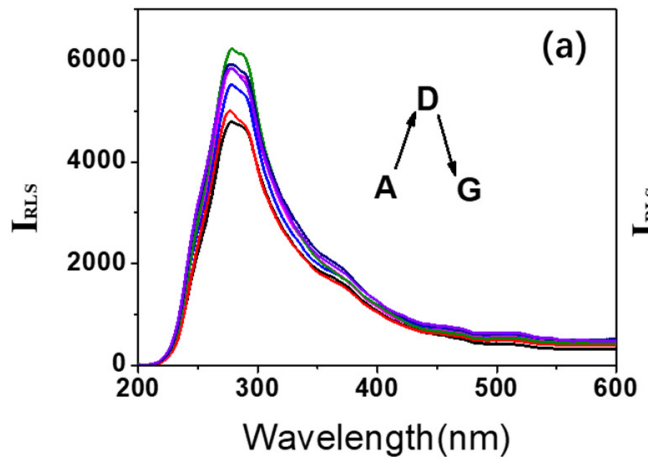

structure of HSA. Therefore, it can be deduced that the mechanism of inhibition of DR 80 on HSA might be due to the changes of structure of HSA.

\subsection{Investigations of impact of DR80 on the RLS spectra of HSA}

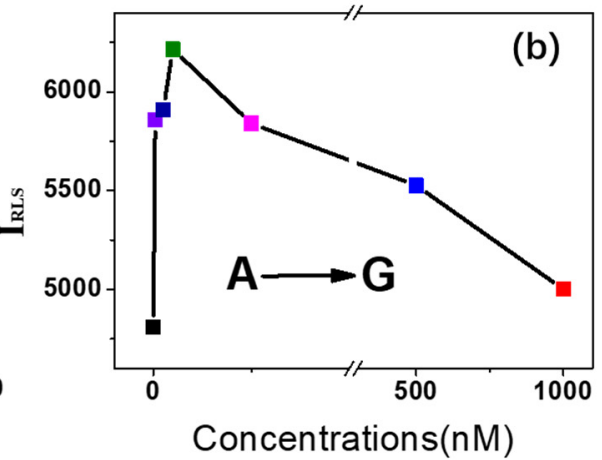

Fig.4 RLS spectra of HSA under treatment of different DR80 concentrations.

(a1) RLS spectra; (b) The scatter plot of RLS spectra intensity in addition of different concentrations of DR80.

Conditions: $\mathrm{T}=310 \mathrm{~K}, \mathrm{pH}=7.4$, [HSA] $=1 \mu \mathrm{M}$

$[\mathrm{DR} 80](\mathrm{A} \rightarrow \mathrm{G})=0,1,5,10,50,500,1000 \mu \mathrm{M}$

Through the above analysis, we can know that DR80 had an effect on the conformation of HSA. Due to the conformation of proteins might be affected by the changes in their particle volume, it is necessary to investigate the protein size after exposure of DR80. Resonance light scattering (RLS) spectroscopy is a reliable tool to evaluate the state of polymerization and detect the hydration diameter change of protein ligand system caused by the combination of protein and ligand.

The value of the RLS spectra increased at 0-10 $\mu \mathrm{M}$ DR80 while then the intensity of RLS decreased with the increasing concentrations of DR80. The enhancement of the RLS intensity at low DR80 concentrations might attribute to the formation of HSA-DR80 agglomerates. Nevertheless, DR80 can destroy the solvent shell on the surface of HSA at high concentrations of DR80, leading to more dispersion of HSA and therefore the smaller particle size of HSA agglomerates. Another possible mechanism for the shrinkage of molecular size was that DR80 at high concentrations led to the "collapse" of protein.

\subsection{Determination of fluorescence quenching mechanism of HSA induced by DR80}
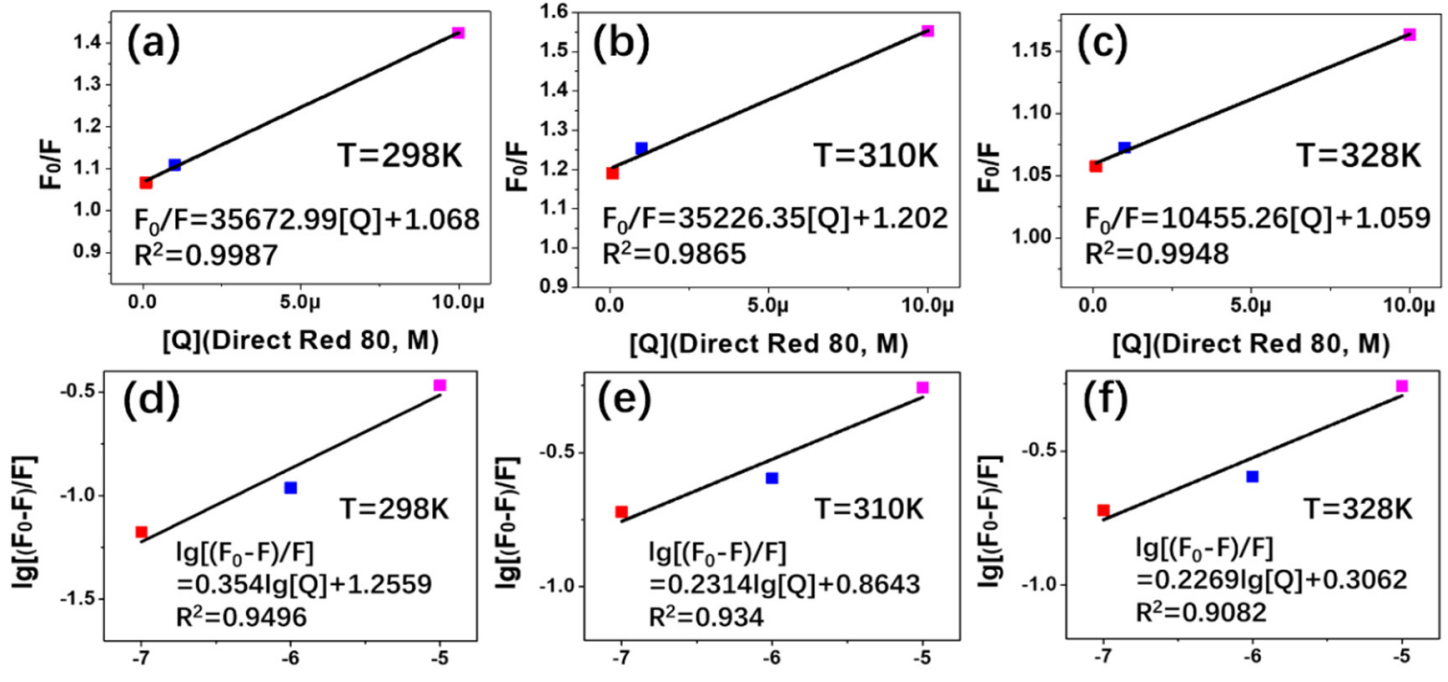

$\lg [Q]$

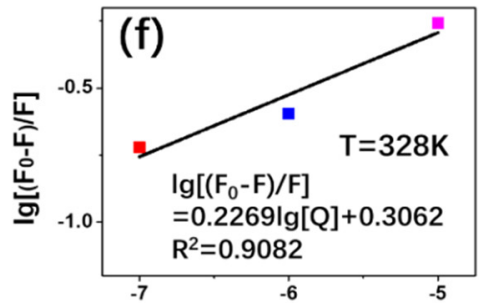

$\lg [Q]$

Fig.5 Plots of Stern-Volmer and $\lg \left[\left(\mathrm{F}_{0}-\mathrm{F}\right) / \mathrm{F}\right]$ vs $\lg [\mathrm{Q}]$ for the fluorescence quenching of HSA induced by DR80

From the discussions at section 3.1.1, we can see that DR80 quenched the endogenous fluorescence of HSA. Therefore, to study the quenching mode, we introduced Stern-Volmer equation[9].

$$
\frac{F_{0}}{F}=K_{S V}[Q]+1=k_{q} \tau_{0}[Q]+1
$$


where $\mathrm{F}_{0}$ and $\mathrm{F}$ are the fluorescence intensities of the fluorophore in the absence and presence of DR80; [Q] is the concentrations of DR80; Ksv is the Stern-Volmer constant and $\mathrm{k}_{\mathrm{q}}$ is the bimolecular quenching rate constant; $\tau_{0}$ is the excited state lifetime of HSA in the absence of quencher (the value of $\tau_{0}$ is $10^{-8} \mathrm{~s}[10]$ ).

Based on the fluorescence data of HSA at 298K, $310 \mathrm{~K}$ and $328 \mathrm{~K}$, the liner curves of $\mathrm{F} / \mathrm{F}_{0}$ versus $\mathrm{Q}$ were established (Fig.5 a-c), and the values of Ksv and Kq were calculated by this equation. The calculated results were displayed in the Table.2. According to Ye-Zhong Zhang's theory, there are two types of quenching: static quenching and static quenching. When the temperature increases, the dynamic quenching constant increases while the static quenching constant decreases. This is mainly because the higher temperature will decrease the stability of the complex and increase the diffusion coefficient. From the Table.2, we can see that the values of Ksv decreased with the increasing temperature. In addition, the $\mathrm{Kq}$ values of the system were much larger than the maximum value of the dynamic quenching rate constant $\left(2.0 \times 10^{10} \mathrm{Lmol}^{-1} \mathrm{~s}^{-1}\right)$ under three temperature conditions. Therefore, all these results confirmed the formation of the DR80-HSA complex, and its quenching type is static quenching.

Table.2 Stern-Volmer quenching constants at different temperatures.

\begin{tabular}{|c|c|c|c|c|}
\hline$T(K)$ & $\begin{array}{c}\mathrm{Ksv} \\
\left(\times 10^{4} \mathrm{Lmol}^{-1}\right)\end{array}$ & $\begin{array}{c}\mathrm{K}_{\mathrm{q}} \\
\left(\times 10^{12} \mathrm{Lmol}^{-1} \mathrm{~s}^{-1}\right)\end{array}$ & ${ }^{a} R^{2}$ & $\begin{array}{c}{ }^{\text {b S.D. }} \\
\left(\times 10^{4} \mathrm{Lmol}^{-1}\right)\end{array}$ \\
\hline 298 & 3.57 & 3.57 & 0.9987 & 0.0928 \\
\hline 310 & 3.52 & 3.52 & 0.9865 & 0.2905 \\
\hline 328 & 1.05 & 1.05 & 0.9948 & 0.0532 \\
\hline
\end{tabular}

$\mathbf{a}: \mathbf{R}^{2}$ is the correlation coefficient.

b:S.D. is the standard deviation for the Ksv.

\subsection{Binding constant and binding capacity between DR80 and HSA}

For static quenching, we introduce the following formula to determine the binding constant.

$$
\lg \frac{\left(F_{0}-F\right)}{F}=\lg K a+n \lg [Q]
$$

where $\mathrm{Ka}$ is the binding constant of HSA and DR80, and the implication of $\mathrm{F}_{0}, \mathrm{~F}$ and $[\mathrm{Q}]$ are the same as in $\mathrm{Eq}$ (3). The calculated results are shown in Table 3.

The $\mathrm{Ka}$ values demonstrating that the interaction between HSA and DR80 was moderate. The decreasing trend of Ka values indicated that a reversible DR80-HSA complex came into being and that it could circulate through the system and serve as a depot of DR80.

Table.3 Binding constants and thermodynamic constants.

\begin{tabular}{|c|c|c|c|c|c|c|}
\hline $\mathbf{T}(\mathbf{K})$ & $\begin{array}{c}\mathrm{Ka} \\
\left(\mathrm{Lmol}^{-1}\right) \\
\end{array}$ & ${ }^{a} \mathbf{R 2}$ & b S.D. & $\Delta \mathbf{H}^{\circ}\left(\mathrm{KJmol}^{-1}\right)$ & $\Delta S^{\circ}\left(\mathrm{Jmol}^{-1} \mathbf{K}^{-1}\right)$ & $\Delta \mathbf{G}^{\circ}\left(\mathrm{KJmol}^{-1}\right)$ \\
\hline 298 & 18.03 & 0.9496 & 0.4938 & & -169.71 & -7.165 \\
\hline 310 & 7.31 & 0.934 & 0.3727 & -57.74 & -169.72 & -5.126 \\
\hline 328 & 2.3 & 0.9082 & 0.4367 & & -169.11 & -2.271 \\
\hline
\end{tabular}

a: $\mathbf{R}^{\mathbf{2}}$ is the correlation coefficient for the Ka values.

b: S.D. is the standard deviation for the Ka.

\subsection{Investigation of the interaction forces type between DR80 and HAS}

Small organic molecule may act on biological macromolecule through four forms of forces: hydrogen bond, hydrophobic force, electrostatic force, and van der Waals force[11]. These types of forces can be judged by thermodynamic constants indirectly: (a) $\triangle \mathrm{H} \circ<0, \triangle \mathrm{S} \circ$ $<0$ : van der Waals force and hydrogen bond; (b) $\triangle \mathrm{H} \circ>$ $0, \triangle \mathrm{S} \circ>0$ : hydrophobic force; (c) $\triangle \mathrm{H} \circ<0, \triangle \mathrm{S} \circ>0$ : electrostatic force. In this study, the values of $\triangle \mathrm{H}^{\circ}, \triangle \mathrm{G}^{\circ}$ and $\triangle \mathrm{S}^{\circ}$ were calculated by the equations below.

$$
\begin{gathered}
\ln \left(\frac{K_{2}}{K_{1}}\right)=\left(\frac{1}{T_{1}}-\frac{1}{T_{2}}\right)\left(\frac{\Delta H^{\circ}}{R}\right) \\
\Delta G^{\bigcirc}=-R T \ln K^{\bigcirc} \\
\Delta G^{\bigcirc}={ }_{\Delta} H^{\bigcirc}-T \Delta S^{\bigcirc}
\end{gathered}
$$

corresponding temperature (Table.2), respectively; $\mathrm{R}$ is the universal gas constant. When the temperature does not change much, $\triangle \mathrm{H}^{\circ}$ can be regarded as a constant.

As can be seen from Table.1, at ambient temperature, the values of $\triangle \mathrm{H}^{\circ}, \triangle \mathrm{S}^{\circ}$ and $\triangle \mathrm{G}^{\circ}$ were $-57.74,-0.168$ and $-7.165 \mathrm{KJmol}^{-1}$, respectively. The negative values of $\triangle \mathrm{H}^{\circ}$ and $\triangle \mathrm{S}^{\circ}$ demonstrating that hydrophobic force and van der Waals force played an important role in the binding process, the this process was exothermic. The negative sign of $\triangle \mathrm{G}^{\circ}$ indicated that combination between DR80 and HSA was spontaneous.

\subsection{Study on the binding site between Direct Red 80 and HSA}

X-ray crystallography studies showed that HSA has a heart-shaped spatial structure, which composed of three homologous $\alpha$-helical domains. Each domain is composed of two subdomains ( $\mathrm{A}$ and $\mathrm{B}$ ), and a hydrophobic cavity is formed between the two subdomains[12]. The docking results of DR80 and HSA are as follows.

where $\mathrm{K}_{1}$ and $\mathrm{K}_{2}$ are the binding constants at the 

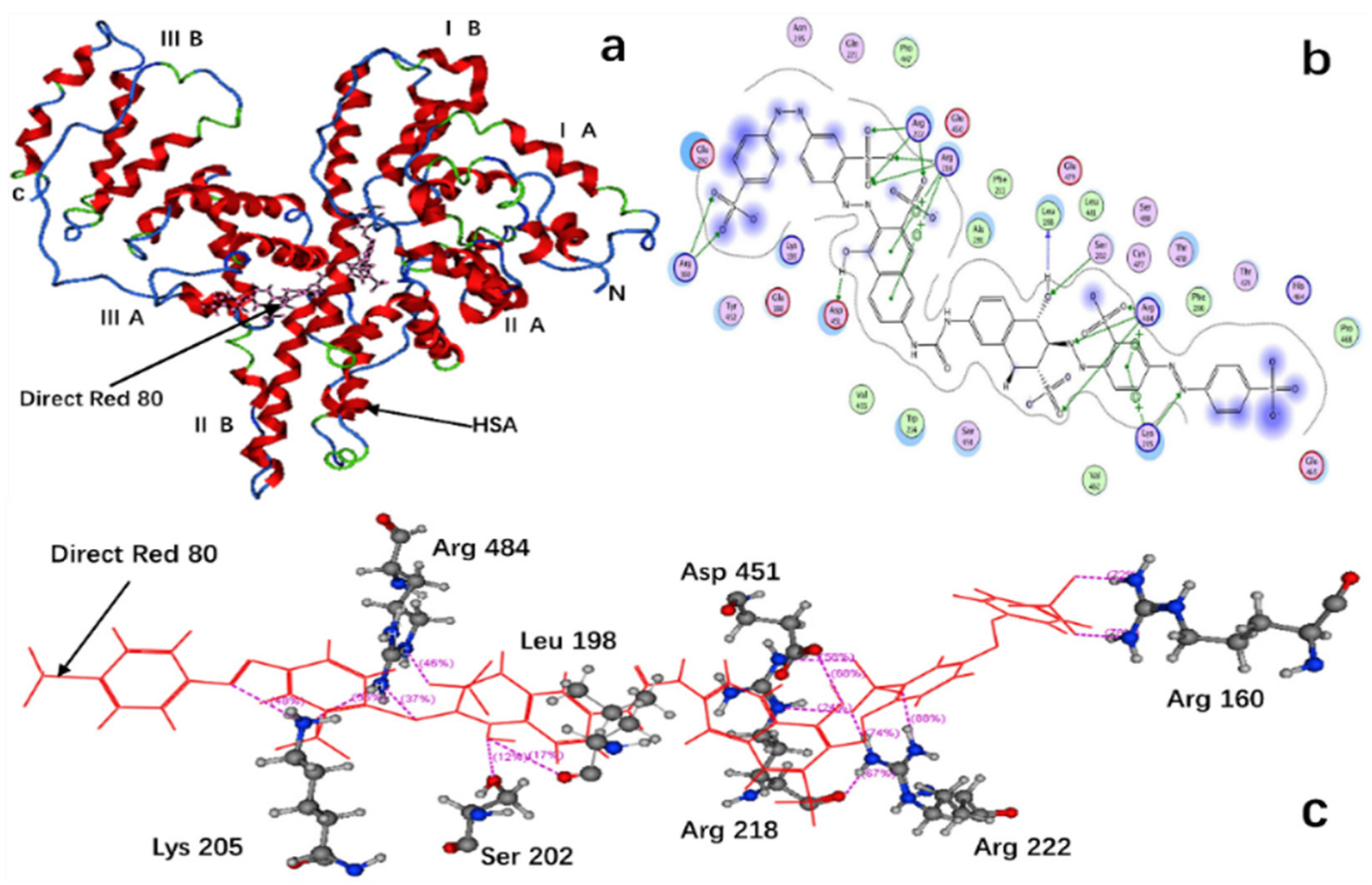

Fig.6 Molecular docking results between DR80 and HSA.

(a) The binding site of DR80 to HSA; (b) The interaction between DR80 and the amino acid residues; (c) Detail docking conformation of DR80 and HAS

Studies have shown that the HSA ligand binding site is mainly located in the hydrophobic cavity of the two structural subdomains of IIA and IIIA, and its site showing esterase activity is located in its structural subdomain IIIA. It can be seen from Fig.6(a) that DR80 bound to the interface of three $\alpha$-helical domains of I, II, and III, rather than the central region of their esterase activity, which further verified that the changes in the HSA skeleton structure and microenvironment caused by DR80 is the main reason of the change in its esterase activity. Fig. $6(\mathrm{~b} / \mathrm{c})$ indicated that the DR80 combined with the HSA in the forms of hydrogen bonds and "arene-cation" force. It is noted that fluorescent chromophore Tyr is located in the binding pocket, demonstrating that DR80 caused impact of the microenvironment of Try.

\section{Conclusions}

In this study, the binding mechanism between DR80 and HSA was explored by multi-spectra, molecular docking, thermomechanical analysis and esterase activity assays. The results indicated that DR80 quenched the endogenous fluorescence of HSA by static quenching and changed the microenvironment of Trp in form of the hydrophobicity increased and the polarity decreased. Thermodynamic analysis results demonstrated that the binding process of DR80 and HSA was spontaneous and exothermic. DR80 bound to the interface of three $\alpha$-helical domains of HSA in the forms of hydrogen bonds and "arene-cation" force, rather than the central region of their esterase activity. Therefore, the changes of structure of HSA was the main reason for the decline of esterase activity. This work provides some valuable information for the interaction between DR80 and HSA, and is helpful for screening the low toxic dye at the molecular level.

\section{Acknowledgements}

This study was supported by the National Key Research and Development Program of China (NO. 2016YFC0402809, 2018YFC0408000, 2018YFC0408006); the Shandong Water Scientific Research and Technology Extension Project (Grant No. SDSLKY201703) and the Technology Demonstration Project of Ministry of Water Resources of China (NO. SF201803).

\section{Reference:}

1. Wells, T.A.; Nakazawa, M.; Manabe, K.; Song, P.S. A conformational change associated with the phototransformation of Pisum phytochrome A as probed by fluorescence quenching. Biochemistry 1994, 33, 708-712.

2. Gomare, S.S.; Jadhav, J.P.; Govindwar, S.P. Degradation of sulfonated azo dyes by the purified lignin peroxidase from Brevibacillus laterosporus MTCC 2298. Biotechnology \& Bioprocess Engineering 2008, 13, 136-143.

3. Hushcha, T.O.; Luik, A.I.; Naboka, Yu.N. Conformation changes of albumin in its interaction with physiologically active compounds as studied by quasi-elastic light scattering spectroscopy and ultrasonic method., doi:10.1016/S00399140(00)00454-9.

4. Yang, F.; Bian, C.; Zhu, L.; Zhao, G.; Huang, Z.; 
Huang, M. Effect of human serum albumin on drug metabolism: structural evidence of esterase activity of human serum albumin. Journal of Structural Biology 2007, 157, 348-355.

5. Arroyo-Maya, I.J.; Campos-Terán, J.; HernándezArana, A.; McClements, D.J. Characterization of flavonoid-protein interactions using fluorescence spectroscopy: Binding of pelargonidin to dairy proteins. Food Chemistry 2016, 213, 431-439, doi:10.1016/j.foodchem.2016.06.105.

6. Bordbar, A.K.; Taherikafrani, A. Binding and fluorescence study on interaction of human serum albumin (HSA) with cetylpyridinium chloride (CPC). Colloids \& Surfaces B Biointerfaces 2007, 55, 84-89.

7. Moghaddam, M.M.; Pirouzi, M.; Saberi, M.R.; Chamani, J. Comparison of the binding behavior of FCCP with HSA and HTF as determined by spectroscopic and molecular modeling techniques: Binding of FCCP to HSA and HTF. Luminescence 2014, 29, 314-331, doi:10.1002/bio.2546.

8. Li, S.; Peng, Z.; Leblanc, R.M. Method To Determine Protein Concentration in the Protein-Nanoparticle Conjugates Aqueous Solution Using Circular Dichroism Spectroscopy. Analytical Chemistry 2015, 87, 6455-6459, doi:10.1021/acs.analchem.5b01451.

9. Paramaguru, G.; Kathiravan, A.; Selvaraj, S.; Venuvanalingam, P.; Renganathan, R. Interaction of anthraquinone dyes with lysozyme: Evidences from spectroscopic and docking studies. Journal of Hazardous Materials 2010, 175, 985-991, doi:10.1016/j.jhazmat.2009.10.107.

10. Lakowicz, J.R.; Weber, G. Quenching of fluorescence by oxygen. Probe for structural fluctuations in macromolecules. Biochemistry 1973, 12, 4161-4170.

11. Leckband, D. Measuring the Forces that Control Protein Interactions. Annu Rev Biophys Biomol Struct 2003, 29, 1-26, doi:10.1146/annurev.biophys.29.1.1.

12. Carter, D.C.; Ho, J.X. Structure of serum albumin. In Advances in protein chemistry; Elsevier, 1994; Vol. 45, pp. 153-203. 